University of Wollongong

Research Online

Australian Institute for Innovative Materials -

Papers

Australian Institute for Innovative Materials

$1-1-2020$

\title{
3D printed soft pneumatic bending sensing chambers for bilateral and remote control of soft robotic systems
}

\author{
Charbel Tawk \\ charbel@uow.edu.au \\ Marc in het Panhuis \\ University of Wollongong, panhuis@uow.edu.au \\ Geoffrey M. Spinks \\ University of Wollongong, gspinks@uow.edu.au \\ Gursel Alici \\ University of Wollongong, gursel@uow.edu.au
}

Follow this and additional works at: https://ro.uow.edu.au/aiimpapers

Part of the Engineering Commons, and the Physical Sciences and Mathematics Commons

\footnotetext{
Research Online is the open access institutional repository for the University of Wollongong. For further information
} contact the UOW Library: research-pubs@uow.edu.au 


\title{
3D printed soft pneumatic bending sensing chambers for bilateral and remote control of soft robotic systems
}

\author{
Abstract \\ (C) 2020 IEEE. This work reports on soft pneumatic bending sensing chambers that are directly 3D printed \\ without requiring any support material and postprocessing using a low-cost and open-source fused \\ deposition modeling (FDM) 3D printer and a commercially available soft thermoplastic polyurethane \\ (TPU). These bending sensing chambers have multiple advantages including very fast response to any \\ change in their internal volume, linearity, negligible hysteresis, repeatability, reliability, stability over time, \\ long lifetime and very low power consumption. The performance of these soft sensing chambers is \\ accurately predicted and optimized using finite element modeling (FEM) and a hyperelastic material \\ model for the TPU used for 3D printing. The soft sensing chambers are tailored to several soft robotic \\ applications such as bending sensors for bilateral control of soft robotic fingers and structures and soft \\ wearable gloves for remote control of soft monolithic robotic fingers and adaptive grippers. \\ Disciplines \\ Engineering | Physical Sciences and Mathematics

\section{Publication Details} \\ Tawk, C., in het Panhuis, P., Spinks, G. \& Alici, G. (2020). 3D printed soft pneumatic bending sensing \\ chambers for bilateral and remote control of soft robotic systems. IEEE/ASME International Conference \\ on Advanced Intelligent Mechatronics, AIM (pp. 922-927).
}




\title{
3D Printed Soft Pneumatic Bending Sensing Chambers for Bilateral and Remote Control of Soft Robotic Systems
}

\author{
Charbel Tawk, Marc in het Panhuis, Geoffrey M. Spinks and Gursel Alici*
}

\begin{abstract}
This work reports on soft pneumatic bending sensing chambers that are directly 3D printed without requiring any support material and postprocessing using a lowcost and open-source fused deposition modeling (FDM) 3D printer and a commercially available soft thermoplastic polyurethane (TPU). These bending sensing chambers have multiple advantages including very fast response to any change in their internal volume, linearity, negligible hysteresis, repeatability, reliability, stability over time, long lifetime and very low power consumption. The performance of these soft sensing chambers is accurately predicted and optimized using finite element modeling (FEM) and a hyperelastic material model for the TPU used for 3D printing. The soft sensing chambers are tailored to several soft robotic applications such as bending sensors for bilateral control of soft robotic fingers and structures and soft wearable gloves for remote control of soft monolithic robotic fingers and adaptive grippers.
\end{abstract}

\section{INTRODUCTION}

Soft robotic concepts and devices are ideal for developing safe human-machine interfaces that are made of highly deformable and soft materials [1]. Recently, diverse soft untethered robots were developed to prove and demonstrate that such robots are more suitable in some robotic areas compared to traditional rigid-bodied robots [2]. Conventional robotic systems are made of stiff and rigid materials and components and therefore cannot operate safely alongside humans and in unstructured environments [3]. Ideally, an entirely soft robot should be made completely of soft and flexible materials that can undergo and sustain large deformations repeatedly [4]. Soft robots require dexterous soft actuators, robust soft sensors and electronics, resilient deformable and flexible structures and compliant power

*This research has been supported by ARC Centre of Excellence for Electromaterials Science (Grant No. CE140100012) and the University of Wollongong, Australia.

C. Tawk is with School of Mechanical, Materials, Mechatronic and Biomedical Engineering, and ARC Centre of Excellence for Electromaterials Science, University of Wollongong, AIIM Facility, NSW, 2522, Australia (e-mail: charbel@uow.edu.au).

G. Alici is with School of Mechanical, Materials, Mechatronic and Biomedical Engineering, and ARC Centre of Excellence for Electromaterials Science, University of Wollongong, AIIM Facility, NSW, 2522, Australia (Corresponding Author, e-mail:gursel@uow.edu.au.)

G. M. Spinks is with the Intelligent Polymer Research Institute, ARC Centre of Excellence for Electromaterials Science, University of Wollongong, AIIM Facility, NSW, 2522, Australia (e-mail: gspinks@uow.edu.au).

M. in het Panhuis is with the School of Chemistry and Molecular Science and Intelligent Polymer Research Institute, ARC Centre of Excellence for Electromaterials Science, University of Wollongong, AIIM Facility, NSW, 2522, Australia (e-mail: panhuis@uow.edu.au).

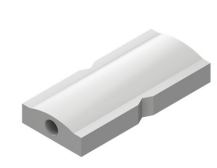

(a)

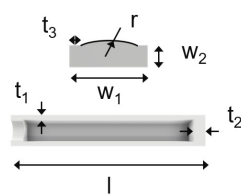

(d)

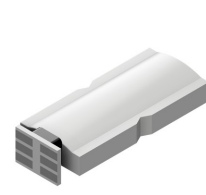

(b)

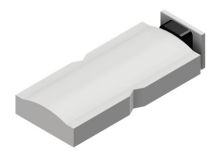

(e)

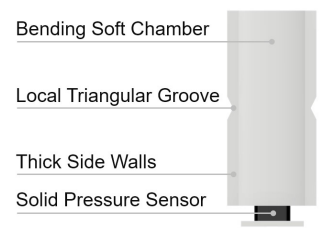

(c)

Upper Thin Curved Wall

Pressure Output Base Thin Wall

(f)
Figure 1. Soft pneumatic bending sensing chambers computer-aided design (CAD) models and dimensions. (a) Soft 3D printed bending chamber. (b) Soft 3D printed bending chamber with the solid air pressure sensor attached. (c) Top view of the assembly. (d) Soft sensor dimensions: 1: $34.0, \mathrm{r}: 15.0, \mathrm{t}_{1}: 0.80, \mathrm{t}_{2}: 2.0, \mathrm{t}_{3}: 3.0, \mathrm{w}_{1}: 15.6, \mathrm{w}_{2}: 4.35$. All dimensions are in $\mathrm{mm}$. (e) Isometric view of the assembly. (f) A cross-sectional view along the length of the soft $3 \mathrm{D}$ printed chamber.

sources (i.e., compliant batteries) [4]. One of the challenges of soft robotics is the development of functional soft and deformable sensors. Soft robotic sensors must be able to sustain large and repeatable deformations and provide acceptable performance. Several resistive strain sensors were developed for soft robots including flex sensors $[5,6]$, conductive inks [7-9], ionic conductive liquids [10], liquid metals [11-13], fabrics and textiles [14, 15], resistive 3D printable thermoplastics [16], soft and elastic compressive foam sensors [17] and ultra-thin piezoresistive sensors [18] combined with 3D printable soft monolithic structures [19]. Also, capacitive soft sensors were developed for pressure sensing [20,21], tactile sensing [22] and strain sensing [23] in several robotic applications. Similarly, strain, curvature, texture and force optical sensors were developed for use in soft prosthetic hands [24]. Some of these sensors have several limitations such as hysteresis, drift, short lifetime or slow response in addition to their laborious manufacturing methods which require multiple fabrication steps before their integration in soft robotic systems.

Also, pneumatic sensing soft structures have been developed for various robotic applications. An air bladder that can be embedded in a shoe to monitor and detect human gait phases was developed by winding a soft silicone tube and connecting it to a pressure sensor [25]. A pneumatic soft sensor for measuring the contact force and curvature in a soft gripper was fabricated using conventional molding and casting techniques that use commercial silicone rubbers [26]. A soft three-axis force sensor, based on radially symmetric 
pneumatic chambers was designed and fabricated by casting silicone rubber [27]. A tactile soft sensor based on a commercially available latex tube that was connected to a pressure sensor was demonstrated for co-operative robots [28]. A method for rapidly prototyping interactive robot skins using 3D printing and analog pressure sensors was presented [29]. Several building blocks were designed to offer different modes of deformation such as bending and twisting. Also, 3D printed pneumatic controls were developed for haptic feedback applications based on the same printing method [30].

These 3D printed soft pneumatic structures were fabricated using high-cost 3D printers and flexible materials with limited performance in terms of deformation. The remaining soft pneumatic structures were fabricated using either commercially available flexible and stretchable silicone tubes or conventional casting and molding techniques involved in developing soft robots [31].

In this work, we present directly $3 \mathrm{D}$ printed soft pneumatic bending sensing chambers that can be rapidly fabricated without requiring support material and postprocessing using a low-cost and open-source fused deposition modeling (FDM) 3D printer and a commercially available soft thermoplastic polyurethane (TPU). These bending soft sensors have multiple advantages including very fast response to any change in their internal volume, linearity, negligible hysteresis, repeatability, reliability, stability over time, long lifetime and very low power consumption. The performance of these sensors can be accurately predicted and optimized using finite element modeling (FEM) and a hyperelastic material model for the TPU used for 3D printing. These soft sensors can be used in diverse soft robotic applications such as bending sensors for bilateral control of soft robotic fingers and structures and soft wearable gloves for remote control of soft adaptive grippers.

\section{MATERIAL AND METHODS}

\section{A. Modeling and Fabrication}

The computer-aided design (CAD) models of the soft pneumatic bending sensing chambers were modeled in Autodesk Fusion 360 (Autodesk Inc.). A commercial slicer (Simplify3D Inc.) was used to slice the CAD models of the bending chambers. The printing parameters were adjusted and optimized in the slicer based on recent studies [32-38] to 3D print airtight soft pneumatic bending sensing chambers. The optimized printing parameters are listed in Table I. The TPU used is NinjaFlex (NinjaTek, USA) which is a commercially available soft material. A low-cost and opensource FDM 3D printer (FlashForge Inventor, FlashForge Corporation) was used to $3 \mathrm{D}$ print the soft bending sensing chambers.

\section{B. TPU Characterization and Material Model}

A uniaxial stress-strain test was performed on the 3D printed TPU to obtain its stress-strain relationship. The tensile tests were conducted on the TPU samples according to the ISO 37 standard where all the samples were stretched by $800 \%$ at a rate of $100 \mathrm{~mm} / \mathrm{s}$ using an electromechanical Instron Universal Testing machine (Instron8801). To assess the effect of the infill pattern on the behavior of the TPU, two different infill patterns, crosswise and longitudinal, were used to $3 \mathrm{D}$ print the test samples. For each type of Infill 8 samples were tested. The infill pattern had an insignificant effect on the behavior of the TPU, as shown in Fig. 2. The TPU was modeled as a hyperelastic material. A 5-Parameter MooneyRivlin material model was implemented based on the average experimental stress-strain data. The parameters of the material model are listed in Table II. This model was implemented in ANSYS Workbench (Release 19.1, ANSYS, Inc.) to perform the FE simulations.

TABLE I. OPTIMIZED PARAMETERS IN SIMPLY3D FOR 3D PRINTING AIRTIGHT AND FUNCTIONAL SOFT PNEUMATIC BENDING SENSING CHAMBERS.

\begin{tabular}{|c|c|c|}
\hline Parameter & Value & Unit \\
\hline \multicolumn{3}{|c|}{ Resolution Settings } \\
\hline Primary Layer Height & 0.1 & $\mathrm{~mm}$ \\
\hline First Layer Height & 0.09 & $\mathrm{~mm}$ \\
\hline First Layer Width & 0.125 & $\mathrm{~mm}$ \\
\hline Extrusion Width & 0.4 & $\mathrm{~mm}$ \\
\hline \multicolumn{3}{|c|}{ Ooze Control } \\
\hline Coast at End & 0.2 & $\mathrm{~mm}$ \\
\hline \multicolumn{3}{|c|}{ Retraction Settings } \\
\hline Retraction Length & 4 & $\mathrm{~mm}$ \\
\hline Retraction Speed & 40 & $\mathrm{~mm} / \mathrm{s}$ \\
\hline \multicolumn{3}{|c|}{ Speed Settings } \\
\hline Default Printing Speed & 10 & $\mathrm{~mm} / \mathrm{s}$ \\
\hline Outline Printing Speed & 8 & $\mathrm{~mm} / \mathrm{s}$ \\
\hline Solid Infill Speed & 8 & $\mathrm{~mm} / \mathrm{s}$ \\
\hline First Layer Speed & 8 & $\mathrm{~mm} / \mathrm{s}$ \\
\hline $\mathrm{X} / \mathrm{Y}$ Axis Movement Speed & 50 & $\mathrm{~mm} / \mathrm{s}$ \\
\hline Z Axis Movement Speed & 20 & $\mathrm{~mm} / \mathrm{s}$ \\
\hline \multicolumn{3}{|c|}{ Temperature Settings } \\
\hline Printing Temperature & 240 & ${ }^{\circ} \mathrm{C}$ \\
\hline Heat Bed Temperature & 32 & ${ }^{\circ} \mathrm{C}$ \\
\hline \multicolumn{3}{|c|}{ Cooling Settings } \\
\hline Fan Speed & 50 & $\%$ \\
\hline \multicolumn{3}{|c|}{ Infill Settings } \\
\hline Infill Percentage & 100 & $\%$ \\
\hline Infill/Perimeter Overlap & 30 & $\%$ \\
\hline \multicolumn{3}{|c|}{ Thin Walls and Movements Behavior } \\
\hline Allowed Perimeter Overlap & 25 & $\%$ \\
\hline External Thin Wall Type & Perimeters Only & - \\
\hline Internal Thin Wall Type & Allow Single Extrusion Fill & - \\
\hline Avoid Crossing Outline & ENABLED & - \\
\hline Detour Factor & 100 & - \\
\hline \multicolumn{3}{|c|}{ Additional Settings } \\
\hline Extrusion Multiplier & 1.15 & - \\
\hline Top Solid Layers & 5 & - \\
\hline Bottom Solid Layers & 5 & - \\
\hline Outline/Perimeter Shells & 25 & - \\
\hline Wipe Nozzle & DISABLED & - \\
\hline Support Material & DISABLED & - \\
\hline
\end{tabular}

TABLE II. OPTIMIZED PARAMETERS IN SIMPLY3D FOR 3D PRINTING AIRTIGHT AND FUNCTIONAL SOFT PNEUMATIC BENDING SENSORS.

\begin{tabular}{|c|c|c|c|}
\hline $\begin{array}{c}\text { Hyperelastic } \\
\text { Material Model }\end{array}$ & Material Constant & Value & Unit \\
\hline \multirow{6}{*}{$\begin{array}{l}\text { 5-Parameter } \\
\text { Mooney Rivlin }\end{array}$} & $\mathrm{C} 10$ & -0.233 & $\mathrm{MPa}$ \\
\hline & $\mathrm{C} 01$ & 2.562 & $\mathrm{MPa}$ \\
\hline & $\mathrm{C} 20$ & 0.116 & $\mathrm{MPa}$ \\
\hline & C11 & -0.561 & $\mathrm{MPa}$ \\
\hline & $\mathrm{C} 02$ & 0.900 & $\mathrm{MPa}$ \\
\hline & $\begin{array}{l}\text { Incompressibility } \\
\text { Parameter D1 }\end{array}$ & 0.000 & $\mathrm{MPa}^{-1}$ \\
\hline
\end{tabular}




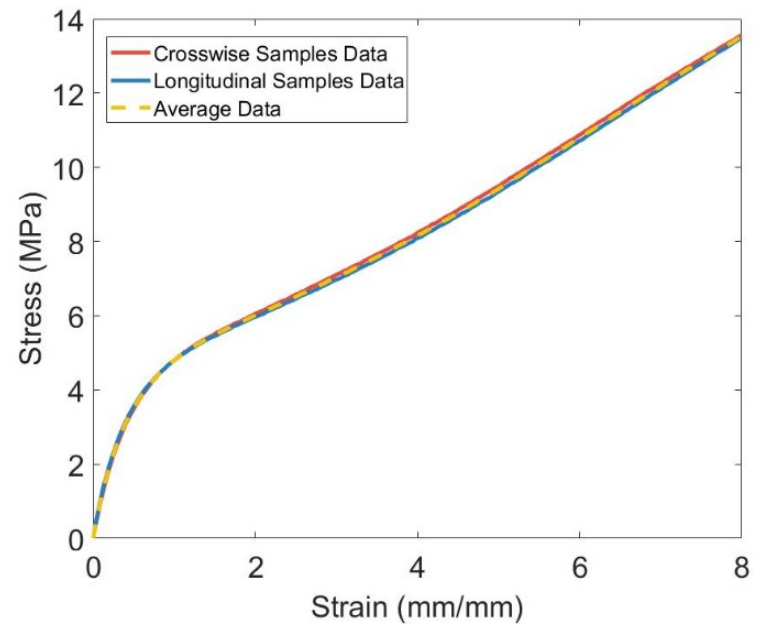

Figure 2. TPU stress-strain data.

\section{Finite Element Modeling}

Finite element simulations were performed on the soft pneumatic bending sensing chambers to optimize their topology in order to predict their behavior under an input mechanical bending load and to obtain a linear relationship between the applied mechanical bending load and the change in their internal volume. The FEM simulations were performed in ANSYS Mechanical where a Static Structural Analysis is implemented. The CAD models were meshed using higher order tetrahedral elements. Contact pairs were defined between the internal walls that come into contact when large mechanical deformations are applied to the bending sensing chambers. In terms of boundary conditions, Fixed Support was defined on one side of the soft structure and a Displacement Support was imposed on their opposite end to simulate the bending deformation applied. The FEM simulations proved that a linear relationship exists between the applied mechanical load and the change in the internal volume of the bending sensor, as shown in Fig. 3. Ideally, a relationship exists between the change in the internal volume of the soft pneumatic bending sensing chamber and the experimental pressure change $\left(\mathrm{P}_{1} \mathrm{~V}_{1}=\mathrm{P}_{2} \mathrm{~V}_{2}\right)$ obtained due to the mechanical deformation applied. Therefore, FEM can be used to predict the behavior of the bending sensing chambers and to optimize their topology to meet specific design requirements.

\section{OBJECTIVE, CONCEPT AND DESIGN}

The objective is to design 3D printed pneumatic bending sensing chambers that can be fabricated using low-cost FDM $3 \mathrm{D}$ printers and commercially available soft materials. The design and dimensions of the chambers are shown in Fig. 1. The geometry of the chambers was optimized using FEM to obtain a linear relationship between the input mechanical bending deformation and the output pressure. The minimum thickness for the thin walls is $0.8 \mathrm{~mm}$. This thickness was used to ensure that the $3 \mathrm{D}$ printed soft sensing chambers are airtight and flexible. Thicker walls can result in a stiffer structure that is harder to bend. The length of the chamber can be chosen depending on the application required. A triangular groove with a base of $4.0 \mathrm{~mm}$ and a height of $1.0 \mathrm{~mm}$ can be added to obtain a local bending joint at a specific position along the length of the sensor, as shown in Fig. 1.

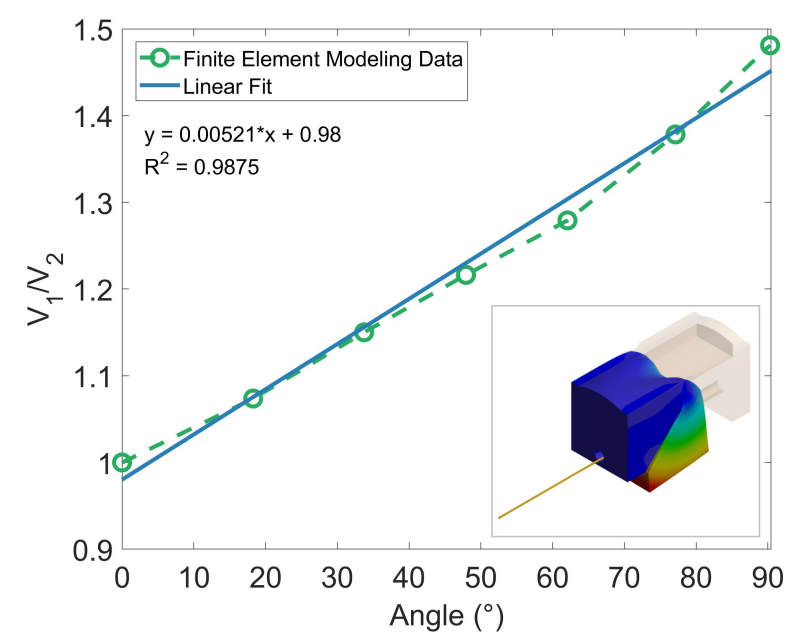

Figure 3. Soft pneumatic bending sensing chamber final design FEM results. Inset: FEM deformed model.

\section{CHARACTERIZATION OF THE SOFt BENDING CHAMBERS}

The experimental setup used to characterize a single soft pneumatic sensing chamber is illustrated in Fig. 4. The bending chamber was printed along with a soft hinge made of the same material. A linear displacement was applied to pull the tendon and consequently bend the soft hinge and thus the chamber by the desired angle.

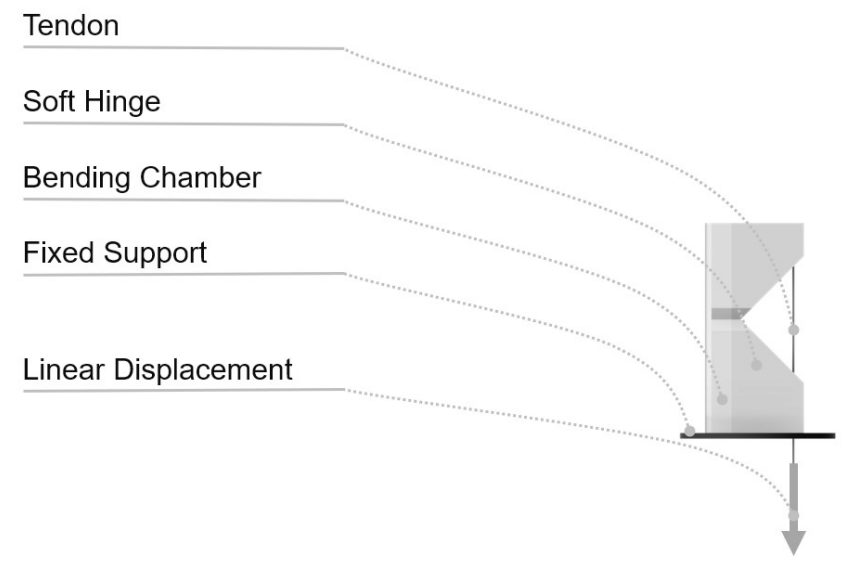

Figure 4. Characterization experimental setup of the soft pneumatic bending sensing chamber.

\section{A. Linearity and Hysteresis}

The linearity and hysteresis of the soft bending sensing chambers were assessed. Fig. 5 shows that the chambers exhibit a linear behavior and have insignificant hysteresis. This proves that these sensing chambers can be used easily and reliably in soft robotic control applications. 


\section{B. Repeatability and Reliability}

The soft bending sensing chamber was activated repeatedly for 500 cycles at a frequency of $1.0 \mathrm{~Hz}$ to assess its repeatability. The sensing chamber generated a repeatable and consistent signal during the activation period, as shown in Fig. 6 and Fig. 7. This proves that these sensing chambers are reliable for use in soft robotic applications.

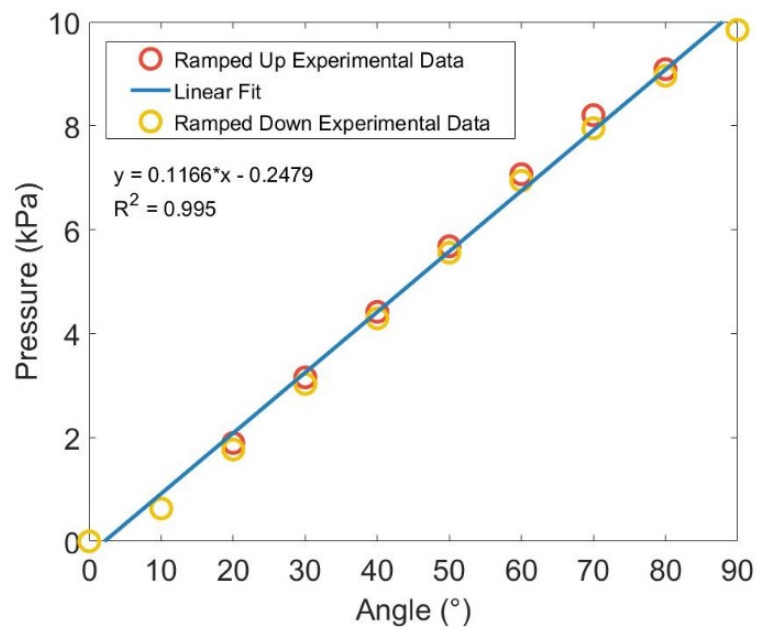

Figure 5. Soft bending sensors linearity and hysteresis data.

\section{Stability Over Time}

The soft bending sensing chamber was bent for 30 minutes where its internal pressure was measured and monitored to assess its stability. Fig. 8 shows that the internal pressure of the soft chamber remained the same during the activation period. This result proved that the sensing chamber generated a stable signal without any drift over time and confirmed that its soft $3 \mathrm{D}$ printed structure is airtight.

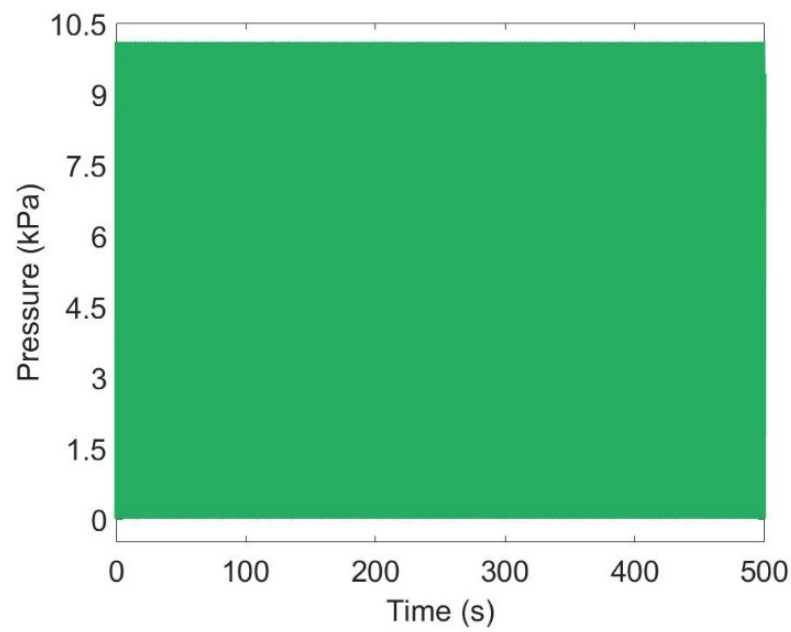

Figure 6. Soft pneumatic bending sensor repeatability signal for 500 cycles at a frequency of $1.0 \mathrm{~Hz}$.

\section{Lifetime}

A single soft bending sensing chamber was repeatably activated to assess its performance in terms of lifetime. The sensor sustained more than 150,000 activation cycles without failure. This result shows that these sensing chambers can be used in soft robotic structures where repeatable deformations are involved for long periods.

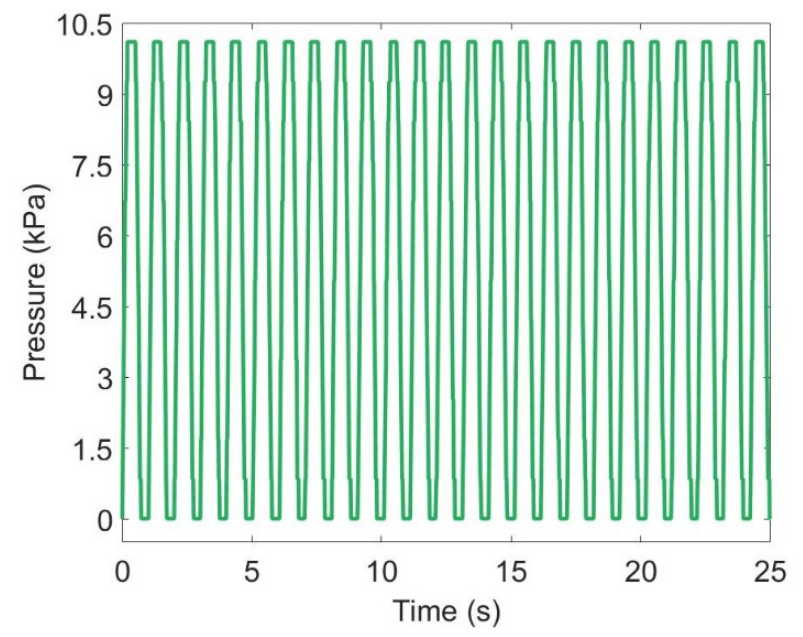

Figure 7. Soft pneumatic bending sensor repeatability signal for 25 cycles at a frequency of $1.0 \mathrm{~Hz}$.

\section{E. Power Consumption and Response Time}

These soft bending sensing chambers are not by themselves sensors. However, solid air pressure sensors are required to sense the volume change upon their deformation. We used commercially available and analog air pressure sensors (ABPDANT015PGAA5, 0-15psi Gauge, 0.25\% accuracy, Honeywell International Inc.) that can be directly connected to the soft bending structure to measure the volume change (i.e., pressure change), as shown in Fig. 1. The power consumption of a single analog pressure sensor is $13.5 \mathrm{~mW}$ [39]. These sensors have a response time of $1.0 \mathrm{~ms}$ [39].

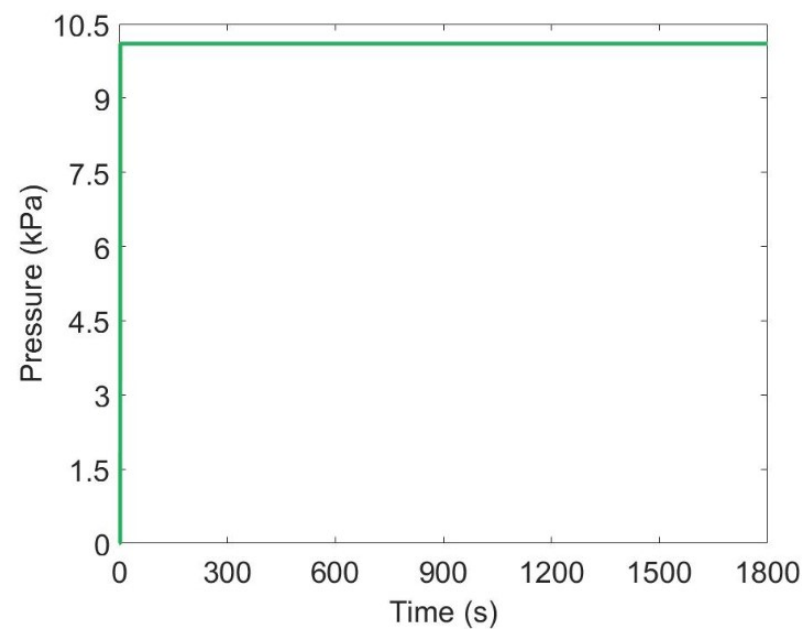

Figure 8. Soft pneumatic bending sensor stability over time (SOT) signal.

\section{ApPlications In SOFT RoBotics}

These soft bending sensing chambers can be tailored to 
diverse soft robotic applications including bending sensors for bilateral control of soft robotic fingers and structures and soft wearable gloves for soft adaptive grippers telecontrol.

\section{A. Robotic Fingers Bilateral Control}

The soft bending sensors can be directly integrated into soft robotic fingers for simple and accurate bilateral control applications. In this scenario, we have 3D printed a soft monolithic robotic finger with three flexural hinges using the same FDM printing method, as shown in Fig. 9. To demonstrate the use of these sensors in bilateral control applications we placed a single bending soft sensing chamber along the back of a soft robotic master finger to detect its position, as shown in Fig. 9. The tendon-driven slave 3D printed monolithic robotic finger is directly connected to a servo motor. There is a direct relationship between the output pressure of the bending sensing chamber placed on the master finger and the servo motor angle. The slave fingers can follow accurately the movements imposed on the master finger, as shown in Fig. 9 and Video S1. The objective of this demonstration was to prove that a single bending sensing chamber can be used for accurate bilateral control.

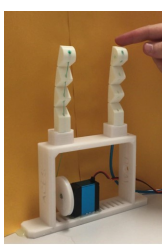

(a)

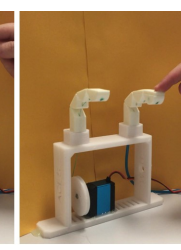

(b)

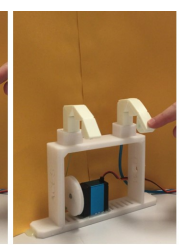

(c)

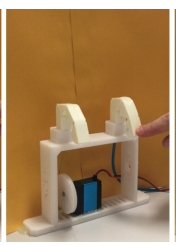

(d)

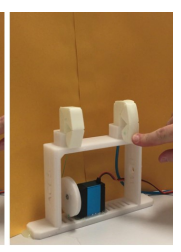

(e)
Figure 9. Soft 3D printed monolithic robotic fingers bilateral control using a master robotic finger (right) and a slave robotic finger (left). (a) The initial position of the robotic fingers. (b) - (e) The slave finger following the same position imposed on the master finger.

\section{B. Soft Wearable Glove for Robotic Fingers Telecontrol}

A soft robotic wearable glove based on five bending sensing chambers was developed for the telecontrol of soft robotic devices. Here, we demonstrate the soft glove using only the index finger to control a soft monolithic robotic finger, as shown in Fig. 10. The soft glove can be used to control the soft robotic finger remotely, as shown in Fig. 10 and Video S1.

\section{Soft Wearable Glove for Soft Grippers Telecontrol}

The same soft wearable glove was used to control a soft adaptive gripper, as shown in Fig. 11 and Video S1. The soft gripper can be controlled remotely with great accuracy and precision using the soft glove to grasp a wide variety of objects with different weights, shapes, sizes, textures and stiffnesses. These gloves can be used to control soft structures remotely.

\section{CONCLUSION}

In this work, we have developed soft pneumatic bending sensing chambers that can be directly manufactured using a low-cost and open-source FDM 3D printer without requiring support material and postprocessing. The soft bending sensing chambers have multiple advantages including very

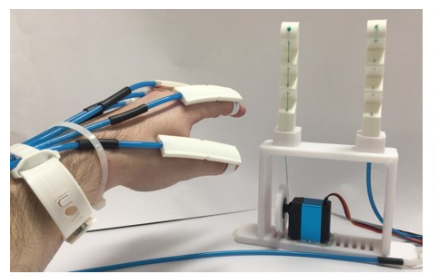

(a)

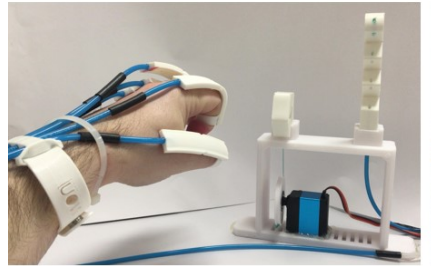

(c)

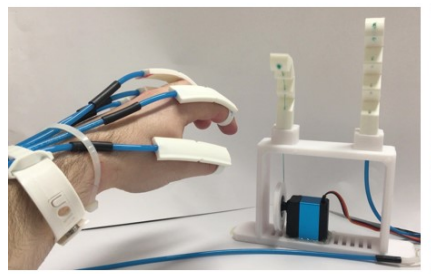

(e)

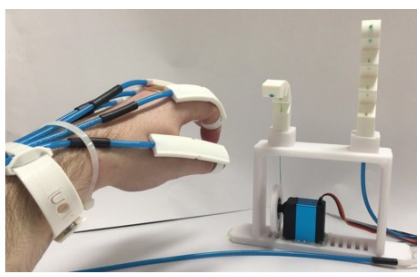

(b)

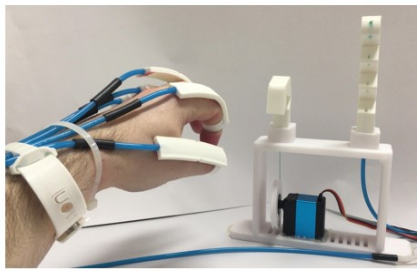

(d)

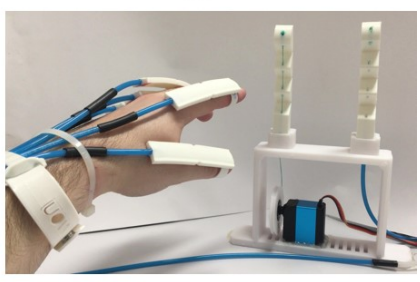

(f)
Figure 10. A Soft 3D printed monolithic robotic finger (left) controlled using the soft wearable glove (index finger only). (a) Initial position. (b) (f) The robotic finger following the same position imposed on the index finger using the soft glove.

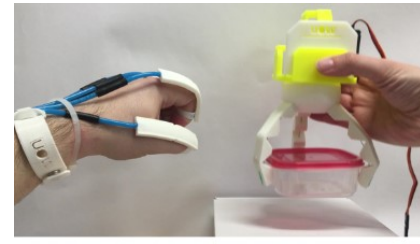

(a)

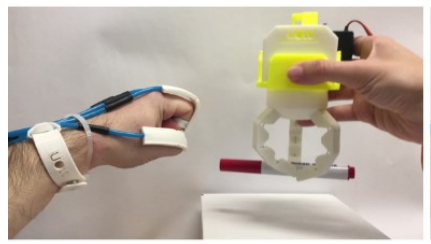

(c)

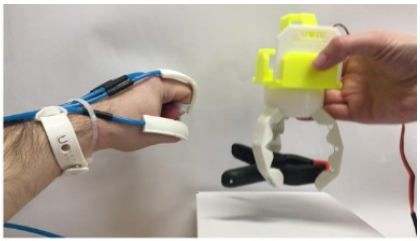

(b)

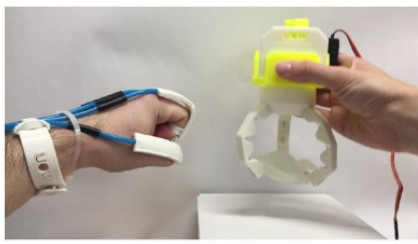

(d)
Figure 11. A soft 3D printed three-finger adaptive gripper controlled using the soft wearable glove. The soft gripper is controlled to pick and place different objects including (a) a small lunch box $(24.04 \mathrm{~g})$, (b) a plastic clamp (41.99g), (c) a marker (10.51g) and (d) a soft and deformable 3D printed robot $(9.99 \mathrm{~g})$.

fast response, linearity, insignificant hysteresis, repeatability, reliability, stability over time, long lifetime and low power consumption. The soft bending chambers were optimized using FEM to obtain a linear relationship between the input mechanical bending deformation and the output pressure.

These soft bending sensing chambers can be used in diverse soft robotic applications including bending sensors for soft robotic fingers and structures bilateral control and soft 
wearable gloves for soft robotic fingers and adaptive grippers telecontrol.

In future work, the soft bending sensing chambers that make the soft wearable glove will be integrated on each joint of the finger to obtain the position of each joint. The soft glove will be developed as a standalone wearable device that can be directly used. Also, for the master finger in the bilateral control application, each of its joints will be assigned a soft bending sensing chamber to demonstrate it with a slave finger that has an actuator on each joint.

\section{ACKNOWLEDGMENT}

The authors acknowledge and thank Rahim Mutlu (University of Wollongong) for his valuable input and suggestions for designing the soft robotic applications demonstrated in this study.

\section{REFERENCES}

[1] C. Majidi, "Soft-Matter Engineering for Soft Robotics," Adv. Mater. Technol, vol. 4, no. 2, p. 1800477, 2019.

[2] M. T. Tolley et al., "A Resilient, Untethered Soft Robot," Soft Robot., vol. 1, no. 3, pp. 213-223, 2014.

[3] B. Trimmer, "Soft robots," Curr. Biol., vol. 23, no. 15, pp. R639R641, 2013.

[4] G. Alici, "Softer is Harder: What Differentiates Soft Robotics from Hard Robotics?," MRS Adv., vol. 3, no. 28, pp. 1557-1568, 2018.

[5] G. Gerboni, A. Diodato, G. Ciuti, M. Cianchetti, and A. Menciassi, "Feedback Control of Soft Robot Actuators via Commercial Flex Bend Sensors," IEEE/ASME Trans. Mech., vol. 22, no. 4, pp. 1881$1888,2017$.

[6] K. Elgeneidy, N. Lohse, and M. Jackson, "Bending angle prediction and control of soft pneumatic actuators with embedded flex sensors A data-driven approach," Mechatronics, vol. 50, pp. 234-247, 2018.

[7] J. T. Muth et al., "Embedded 3D Printing of Strain Sensors within Highly Stretchable Elastomers," Adv. Mater., vol. 26, no. 36, pp. 6307-6312, 2014.

[8] J. C. Yeo, H. K. Yap, W. Xi, Z. Wang, C.-H. Yeow, and C. T. Lim, "Flexible and Stretchable Strain Sensing Actuator for Wearable Soft Robotic Applications," Adv. Mater. Technol., vol. 1, no. 3, p. $1600018,2016$.

[9] S. Kumbay Yildiz, R. Mutlu, and G. Alici, "Fabrication and characterisation of highly stretchable elastomeric strain sensors for prosthetic hand applications," Sens. Actuators, A, vol. 247, pp. 514$521,2016$.

[10] R. L. Truby et al., "Soft Somatosensitive Actuators via Embedded 3D Printing," Adv. Mater., vol. 30, no. 15, p. 1706383, 2018.

[11] Y. Park, B. Chen, and R. J. Wood, "Design and Fabrication of Soft Artificial Skin Using Embedded Microchannels and Liquid Conductors," IEEE Sens. J., vol. 12, no. 8, pp. 2711-2718, 2012.

[12] W. Xi, J. C. Yeo, L. Yu, S. Zhang, and C. T. Lim, "Ultrathin and Wearable Microtubular Epidermal Sensor for Real-Time Physiological Pulse Monitoring," Adv. Mater. Technol., vol. 2, no. 5, p. 1700016, 2017.

[13] M. D. Dickey, "Stretchable and Soft Electronics using Liquid Metals," Adv. Mater., vol. 29, no. 27, p. 1606425, 2017.

[14] M. Stoppa and A. Chiolerio, "Wearable Electronics and Smart Textiles: A Critical Review," Sensors, vol. 14, no. 7, p. 11957, 2014.

[15] M. C. Lina and B. F. Alison, "Smart fabric sensors and e-textile technologies: a review," Smart Mater. Struct., vol. 23, no. 5, p. 053001, 2014.

[16] K. Elgeneidy, G. Neumann, M. Jackson, and N. Lohse, "Directly Printable Flexible Strain Sensors for Bending and Contact Feedback of Soft Actuators," Front. Robot. AI, vol. 5, no. 2, 2018.

[17] V. Sencadas, C. Tawk and G. Alici, "Highly Sensitive Soft Foam Sensors to Empower Robotic Systems," Adv. Mater. Technol., p. $1900423,2019$.
[18] H. Mai, R. Mutlu, C. Tawk, G. Alici, and V. Sencadas, "Ultrastretchable MWCNT-Ecoflex piezoresistive sensors for human motion detection applications," Compos. Sci. Technol., vol. 173, pp. 118-124, 2019.

[19] V. Sencadas, R. Mutlu, and G. Alici, "Large area and ultra-thin compliant strain sensors for prosthetic devices," Sens. Actuators, A, vol. 266 , pp. 56-64, 2017.

[20] O. Atalay, A. Atalay, J. Gafford, and C. Walsh, "A Highly Sensitive Capacitive-Based Soft Pressure Sensor Based on a Conductive Fabric and a Microporous Dielectric Layer," Adv. Mater. Technol., vol. 3, no. 1, p. $1700237,2018$.

[21] L. Viry et al., "Flexible Three-Axial Force Sensor for Soft and Highly Sensitive Artificial Touch," Adv. Mater., vol. 26, no. 17, pp. 26592664, 2014.

[22] L. Bin, G. Yang, F. Adam, and V. Yon, "Soft capacitive tactile sensing arrays fabricated via direct filament casting," Smart Mater. Struct., vol. 25, no. 7, p. 075009, 2016.

[23] A. Frutiger et al., "Capacitive Soft Strain Sensors via Multicore-Shell Fiber Printing," Adv. Mater., vol. 27, no. 15, pp. 2440-2446, 2015.

[24] H. Zhao, K. O’Brien, S. Li, and R. F. Shepherd, "Optoelectronically innervated soft prosthetic hand via stretchable optical waveguides," Sci. Robot., vol. 1, no. 1, 2016.

[25] K. Kong and M. Tomizuka, "A Gait Monitoring System Based on Air Pressure Sensors Embedded in a Shoe," IEEE/ASME Trans. Mech., vol. 14, no. 3, pp. 358-370, 2009.

[26] H. Yang, Y. Chen, Y. Sun, and L. Hao, "A novel pneumatic soft sensor for measuring contact force and curvature of a soft gripper," Sens. Actuators, A, vol. 266, pp. 318-327, 2017.

[27] H. Choi, P. Jung, K. Jung, and K. Kong, "Design and fabrication of a soft three-axis force sensor based on radially symmetric pneumatic chambers," in Proc. IEEE Int. Conf. Robot. Autom., 2017, pp. 55195524.

[28] D. Gong, R. He, J. Yu, and G. Zuo, "A Pneumatic Tactile Sensor for Co-Operative Robots," Sensors, vol. 17, no. 11, p. 2592, 2017.

[29] R. Slyper and J. Hodgins, "Prototyping robot appearance, movement, and interactions using flexible 3D printing and air pressure sensors," in Proc. IEEE RO-MAN: The 21st IEEE International Symposium on Robot and Human Interactive Communication, 2012, pp. 6-11.

[30] M. Vázquez et al., "3D Printing Pneumatic Device Controls with Variable Activation Force Capabilities," in Proc. of the 33rd Annual ACM Conference on Human Factors in Computing Systems, 2015, pp. 1295-1304.

[31] A. D. Marchese, R. K. Katzschmann, and D. Rus, "A Recipe for Soft Fluidic Elastomer Robots," Soft Robot., vol. 2, no. 1, pp. 7-25, 2015.

[32] C. Tawk, M. in het Panhuis, G.M, Spinks, and G. Alici, "Bioinspired 3D Printable Soft Vacuum Actuators (SOVA) for Locomotion Robots, Grippers and Artificial Muscles", Soft Robot., vol. 5, no. 6, pp. 685694, 2018.

[33] C. Tawk, M. in het Panhuis, G. M. Spinks, and G. Alici, "Soft Pneumatic Sensing Chambers for Generic and Interactive HumanMachine Interfaces," Adv. Intell. Sys., vol. 1, no. 1, p. 1900002, 2019.

[34] R. Mutlu, C. Tawk, G. Alici, and E. Sariyildiz, "A 3D printed monolithic soft gripper with adjustable stiffness," in Proc. IECON 2017 - 43rd Annual Conference of the IEEE Industrial Electronics Society, 2017, pp. 6235-6240.

[35] C. Tawk, G.M. Spinks, M. in het Panhuis, and G. Alici, "3D Printable Linear Soft Vacuum Actuators (LSOVA): their modeling, performance quantification and application in soft robotic systems," IEEE/ASME Trans. Mech. DOI: 10.1109/TMECH.2019.2933027, 2019.

[36] C. Tawk, G.M, Spinks, M. in het Panhuis, and G. Alici, "3D Printable Vacuum-Powered Soft Linear Actuators," in Proc. IEEE/ASME Int. Conf. Adv. Intell. Mech. 2019, pp. 50-55.

[37] C. Tawk, Y. Gao, R. Mutlu, and G. Alici, "Fully 3D Printed Monolithic Soft Gripper with High Conformal Grasping Capability," in Proc. IEEE/ASME Int. Conf. Adv. Intell. Mech. 2019, pp. 11391144.

[38] C. Tawk, A. Gillett, M. in het Panhuis, G.M, Spinks, and G. Alici, "A 3D-Printed Omni-Purpose Soft Gripper", IEEE Trans. Robot., DOI: 10.1109/TRO.2019.2924386, 2019.

[39] Honeywell Sensing and Internet of Things, https://sensing.honeywell.com/abpdant015pgaa5-amplified-boardmount-pressure-sensors, accessed July 15, 2019. 